\title{
CONTROLLABILITY OF NONLINEAR DISCRETE SYSTEMS
}

\author{
JERZY KLAMKA* \\ * Institute of Automatic Control, Silesian University of Technology \\ ul. Akademicka 16, 44-100 Gliwice, Poland \\ e-mail: jklamka@ia.polsl.gliwice.pl
}

\begin{abstract}
Local constrained controllability problems for nonlinear finite-dimensional discrete 1-D and 2-D control systems with constant coefficients are formulated and discussed. Using some mapping theorems taken from nonlinear functional analysis and linear approximation methods, sufficient conditions for constrained controllability in bounded domains are derived and proved. The paper extends the controllability conditions with unconstrained controls given in the literature to cover both 1-D and 2-D nonlinear discrete systems with constrained controls.
\end{abstract}

Keywords: nonlinear systems, discrete systems, 2-D systems, controllability, nonlinear covering operators

\section{Introduction}

At the end of the 1960s, the state-space theory of control systems for both time-invariant and time-varying dynamical systems was essentially worked out. The basic concepts of controllability and observability and the weaker notions of stabilizability and detectability play a fundamental role in the solutions of many important different optimal control problems. The primary concern of the present paper is the constrained controllability problem for nonlinear, finite-dimensional, time-invariant discretetime 1-D and 2-D control systems defined in bounded domains.

Controllability is one of the fundamental concepts in modern mathematical control theory. Roughly speaking, controllability means that it is possible to steer a dynamical system from an arbitrary initial state to an arbitrary final state using the set of admissible controls. In the literature there are many different definitions of controllability which, strongly depend on the class of dynamical systems considered (Klamka, 1991b; 1993; 1995).

Up to the present time, the problem of controllability in continuous and discrete-time linear dynamical systems has been extensively investigated in many papers (see, e.g., (Klamka, 1991b; 1993; 1995) for an extensive list of publications). However, this is not generally true for nonlinear dynamical systems, especially those with constrained controls. Only several papers concern constrained controllability problems for continuous or discrete nonlinear or linear dynamical systems.

Therefore, in the present paper local constrained controllability problems for nonlinear finite-dimensional discrete 1-D and 2-D systems with constant coefficients are formulated and discussed. Using some mapping theorems from nonlinear functional analysis (Graves, 1950; Robinson, 1986) and linear approximation methods, sufficient conditions for constrained controllability in bounded domains are derived and proved.

Different controllability problems for discrete-time linear dynamical systems with several independent variables (2-D systems or generally $M$-D systems) have been extensively investigated in many papers (see, e.g., (Klamka, 1991b) for a list of publications on this topic). However, it should be pointed out that this is not quite true for nonlinear discrete dynamical 2-D or $M$-D systems, especially those with constrained values of controls. Only several papers concern constrained controllability problems for discrete 2-D or $M$-D nonlinear or linear dynamical systems (Klamka, 1988a; 1992; 1994; 1995). In the paper (Klamka; 1988b) the relationships between local and global controllability for linear 2-D systems with control values in a given neighbourhood of zero are investigated. The paper (Klamka, 1992) contains results concerning the local controllability of nonlinear 2-D systems without differentiability assumptions. In (Klamka, 1992) the global controllability of linear 2-D systems with controls taking their values in a given cone is discussed. Finally, the paper (Klamka, 1995) concerns the local controllability of nonlinear continuous-time dynamical systems.

Summarizing, in the present paper local constrained controllability problems for nonlinear finite-dimensional discrete 1-D and 2-D systems with constant coefficients are formulated and discussed. Using some mapping theorems taken from the theory of nonlinear functional analysis (Graves, 1950; Robinson, 1986) and linear approximation methods (Klamka, 1992; 1995), some sufficient 
conditions for local constrained controllability in bounded domains are derived and proved.

The present paper extends in some sense the results given in the papers (Klamka, 1991a; 1992; 1993; 1995) to cover nonlinear discrete 1-D and 2-D systems with constrained controls.

\section{Preliminaries}

Let us consider a general nonlinear discrete 1-D system with constant coefficients described by the following vector difference equation:

$$
x(i+1)=f(x(i), u(i)),
$$

where $i \in \mathbb{Z}^{+}, \mathbb{Z}^{+}$is the set of non-negative integers, $x(i) \in \mathbb{R}^{n}$ is a state vector at the point $i, u(i) \in \mathbb{R}^{m}$ is a control vector at the point $i$, and $f: \mathbb{R}^{n} \times \mathbb{R}^{m} \rightarrow \mathbb{R}^{n}$ is a given function.

Let $U \subset \mathbb{R}^{m}$ be a given arbitrary set. The sequence of controls $u_{p}=\{u(i) ; 0 \leq i<p, u(i) \in U\}$ is called an admissible sequence of controls. The set of all such admissible sequences of controls forms the so-called admissible set of controls $U_{p}$. In the sequel, we shall also use the following notation: $\Omega^{0} \subset \mathbb{R}^{m}$ is a neighbourhood of zero, $U^{c} \subset \mathbb{R}^{m}$ is a closed convex cone with vertex at zero and $U^{c 0}=U^{c} \cap \Omega^{0}$.

The initial condition for the nonlinear vector difference equation (1) is given by

$$
x(0)=x_{0} \in \mathbb{R}^{n},
$$

where $x_{0}$ is a known vector. For a given initial condition (2) and for an arbitrary admissible sequence of controls, there exists a unique solution of the nonlinear difference equation (1), which may be computed by successive iterations.

Instead of the nonlinear discrete system (1), we shall also consider the associated linear discrete system with constant coefficients described by the vector difference equation

$$
x(i+1)=A x(i)+B u(i)
$$

defined for $i \geq 0$, where $A$ and $B$ are $n \times n$ - and $n \times m$ dimensional constant matrices, respectively.

For the linear discrete system (3) we can define the socalled transition matrix $A^{i}$. Using $A^{i}$, we can express the solution $x(i)$ of (3) for $i>0$ in the following compact form:

$$
x(i)=A^{i} x_{0}+\sum_{j=0}^{j=i-1} A^{i-j-1} B u(j) .
$$

For the zero initial condition $x(0)=x_{0}=0$, the solution $x(i)$ to (3) for $i>0$ is given by

$$
x(i)=\sum_{j=0}^{j=i-1} A^{i-j-1} B u(j) .
$$

Let us introduce the controllability matrix $W_{p}$ for the linear discrete system (3) (Klamka, 1991b; 1993; 1995) defined as follows:

$$
W_{p}=\left[A^{p-1} B, A^{p-2} B, \ldots, A^{i} B, \ldots, A B, B\right] .
$$

Moreover, let the cone $V_{p}^{c} \subset \mathbb{R}^{n}$ denote the image of the cone of admissible controls $U_{p}^{c}$ under the linear mapping $W_{p}: \mathbb{R}^{p m} \rightarrow \mathbb{R}^{n}$ and $V_{p}^{c *} \subset \mathbb{R}^{n}$ denote the so-called polar cone defined as follows:

$$
V_{p}^{c *}=\left\{x^{*} \in \mathbb{R}^{n}:\left\langle x^{*}, x\right\rangle \leq 0 \text { for all } x \in V_{p}^{c}\right\} .
$$

For linear and nonlinear discrete systems it is possible to define many different concepts of controllability, in much the same way as for linear discrete systems. In the sequel, we shall concentrate on local and global $U$ controllabilities in a given interval $[0, p]$.

Definition 1. The system (1) is said to be globally $U$ controllable in a given interval $[0, p]$ if for the zero initial condition $x_{0}=0$, and every vector $x^{\prime} \in \mathbb{R}^{n}$, there exists an admissible sequence of controls $u_{p}=\{u(i) \in$ $U ; \quad 0 \leq i<p\}$ such that the corresponding solution of (1) satisfies the condition $x(p)=x^{\prime}$.

Definition 2. The system (1) is said to be locally $U$ controllable in a given interval $[0, p]$ if for the zero initial condition $x_{0}=0$ there exists a neighbourhood of zero $D \subset \mathbb{R}^{n}$ such that for every point $x^{\prime} \in D$ there exists an admissible sequence of controls $u_{p}=\{u(i) \in U$ : $0 \leq i<p\}$ such that the corresponding solution of (1) satisfies the condition $x(p)=x^{\prime}$.

Of course, the same definitions are valid for the linear discrete system (3).

Now, we shall recall fundamental criteria for global $U^{c}$-controllability, global $\mathbb{R}^{m}$-controllability and local $\Omega^{0}$-controllability in a given interval $[0, p]$ for the linear discrete system (3).

Theorem 1. (Klamka, 1995). The linear discrete system (3) is globally $U^{c}$-controllable in the interval $[0, p]$ if and only if the following two conditions hold:

$$
\begin{gathered}
\operatorname{rank} W_{p}=n, \\
V_{p}^{c *}=\{0\} .
\end{gathered}
$$

From Theorem 1 we obtain directly the necessary and sufficient condition for the global controllability with 
unconstrained controls formulated in the following corollary:

Corollary 1. (Klamka, 1993) The linear discrete system (3) is globally $\mathbb{R}^{m}$-controllable in the interval $[0, p]$ if and only if the condition (7) holds.

It is well known (Klamka, 1995) that for the sets $U$ containing zero as an interior point, the local constrained controllability is equivalent to the global unconstrained controllability.

Corollary 2. (Klamka, 1995) The linear discrete system (3) is locally $\Omega^{0}$-controllable in the interval $[0, p]$ if and only if it is globally $\mathbb{R}^{m}$-controllable in the interval $[0, p]$.

Corollary 2 directly follows from the well-known fact that the range of a bounded linear operator covers the whole space if and only if this operator transforms some neighbourhood of zero onto some neighbourhood of zero in the range space (Graves, 1950).

\section{Main Results}

In this section we shall formulate and prove sufficient conditions of the local $U$-controllability in a given interval $[0, p]$ and different sets $U$ for the nonlinear discrete system (1).

It is generally assumed that

1. $f(0,0)=0$,

2. the function $f(x, u)$ is continuously differentiable with respect to all its arguments in some neighbourhood of zero in the product space $\mathbb{R}^{n} \times \mathbb{R}^{m}$.

Taking into account Assumption 2, let us introduce the following notation for the partial derivatives of $f(x, u)$ :

$$
A=f_{x}^{\prime}(0,0), \quad B=f_{u}^{\prime}(0,0),
$$

where $A$ and $B$ are $n \times n$ - and $n \times m$-dimensional constant matrices, respectively.

Therefore, using standard methods, it is possible to construct a linear approximation of the nonlinear discrete system (1). This linear approximation is valid in some neighbourhood of the point zero in the product space $\mathbb{R}^{n} \times \mathbb{R}^{m}$, and is given by the linear difference equation (3) with the matrices $A, B$ defined above. The proofs of the main results are based on some lemmas from functional analysis concerning the so-called nonlinear covering operators (Graves, 1950; Robinson, 1986). Now, for convenience, we shall shortly state those results.

Lemma 1. (Robinson, 1986). Let $F: Z \rightarrow Y$ be $a$ nonlinear operator from a Banach space $Z$ into a $B a$ nach space $Y$ and suppose that $F(0)=0$. Assume that the Fréchet derivative $\mathrm{d} F(0)$ maps a closed convex cone $C \subset Z$ with vertex at zero onto the whole space $Y$. Then there exist neighbourhoods $M_{0} \subset Z$ around $0 \in Z$ and $N_{0} \subset Y$ around $0 \in Y$ such that the equation $y=F(z)$ has at least one solution $z \in M_{0} \cap C$ for each $y \in N_{0}$.

Let us observe that a direct consequence of Lemma 1 is the following result concerning nonlinear covering operators:

Lemma 2. (Graves, 1950) Let $F: Z \rightarrow Y$ be a nonlinear operator from a Banach space $Z$ into a Banach space $Y$ which has the Fréchet derivative $\mathrm{d} F(0): Z \rightarrow Y$ whose image coincides with the whole space $Y$. Then the image of the operator $F$ will contain a neighbourhood of the point $F(0) \in Y$.

Now, we are in a position to formulate and prove the main result on the local $U$-controllability in the interval $[0, p]$ for the nonlinear discrete system (1).

Theorem 2. Suppose that $U^{c} \subset \mathbb{R}^{m}$ is a closed convex cone with vertex at zero. Then the nonlinear discrete system (1) is locally $U^{c 0}$-controllable in the interval $[0, p]$ if its linear approximation near the origin given by the difference equation (3) is globally $U^{c}$-controllable in the same interval $[0, p]$.

Proof. The proof of Theorem 2 is based on Lemma 1. Let our nonlinear operator $F$ transform the space of admissible control sequence $\{u(i): 0 \leq i \leq p\}$ into the space of solutions at the point $p$ for the nonlinear discrete system (1). More precisely, the nonlinear operator $F: \mathbb{R}^{m} \times \mathbb{R}^{m} \times \cdots \times \mathbb{R}^{m} \rightarrow \mathbb{R}^{n}$ is defined as follows:

$$
\begin{array}{r}
F\{u(0,0), u(1), u(2), \ldots, u(i), \ldots, u(p-1)\} \\
=x(p),
\end{array}
$$

where $x(p)$ is the solution at the point $p$ of the nonlinear discrete system (1) corresponding to an admissible sequence of controls $u_{p}=\{u(i): 0 \leq i<p\}$ and for the zero initial condition. The Fréchet derivative of the nonlinear operator $F$ at zero, denoted by $\mathrm{d} F(0)$, is a bounded linear operator defined by

$$
\begin{array}{r}
\mathrm{d} F(0)\{u(0), u(1), u(2), \ldots, u(i), \ldots, u(p-1)\} \\
=x(p),
\end{array}
$$

where $x(p)$ is the solution at the point $p$ of the linear system (3) corresponding to an admissible sequence of controls $u_{p}=\{u(i): 0 \leq i<p\}$ for the zero initial condition.

Since $f(0,0)=0$, for the zero initial condition the nonlinear operator $F$ transforms zero into zero, i.e. $F(0)=0$. If the linear system (3) is globally $U^{c_{-}}$ controllable in the interval $[0, p]$, then the image of then 
Fréchet derivative $\mathrm{d} F(0)$ covers the whole space $\mathbb{R}^{n}$. Therefore, by the result stated at the beginning of the proof, the nonlinear operator $F$ covers some neighbourhood of zero in the space $\mathbb{R}^{n}$. Hence, by Definition 2 , the nonlinear discrete system (1) is locally $U^{c}$-controllable in the interval $[0, p]$.

Corollary 3. Under the assumptions of Theorem 2, the nonlinear system (1) is locally $U^{c 0}$-controllable in the interval $[0, p]$ if the relations (7) and (8) hold.

If the relations (7) and (8) hold, then by Theorem 1 the linear discrete system (1) is globally $U^{c}$-controllable in the interval $[0, p]$. Therefore, by Theorem 2 , the nonlinear system (1) is locally $U^{c 0}$-controllable in the same interval $[0, p]$. In the case when the set $U$ contains zero as an interior point, we have the following sufficient condition for the local constrained controllability of the nonlinear discrete system (1):

Corollary 4. Let $0 \in \operatorname{int}(U)$. Then the nonlinear discrete system (1) is locally $U$-controllable in the interval $[0, p]$ if its linear approximation near the origin, given by the difference equation (3), is locally $U$-controllable in the same interval $[0, p]$.

As a simple illustrative example, consider the nonlinear discrete system described by the following set of nonlinear difference equations:

$$
\begin{aligned}
& x_{1}(i+1)=x_{1}(i)+x_{1}^{2}(i)+2 x_{2}(i)-u(i)+u^{2}(i), \\
& x_{2}(i+1)=x_{1}^{2}(i)-x_{2}(i)+x_{2}^{2}(i)+u(i),
\end{aligned}
$$

defined in the interval $[0,2]$. Hence $n=2, m=1$ and $p=2$. Let us additionally assume that $U^{c}=\{u \in$ $\mathbb{R}: u \geq 0\}$, i.e. the admissible scalar controls $u(i), i=$ $0,1,2$ are non-negative.

The linear approximation near the origin of the nonlinear discrete system (10) has the form (3) with the matrices

$$
A=\left[\begin{array}{cc}
1 & 2 \\
0 & -1
\end{array}\right], \quad B=\left[\begin{array}{c}
-1 \\
1
\end{array}\right]
$$

Therefore the $2 \times 2$-dimensional controllability matrix $W_{p}=W_{2}$ for the linear approximation has the following form:

$$
W_{2}=\left[\begin{array}{cc}
1 & -1 \\
-1 & 1
\end{array}\right]
$$

Hence $\operatorname{rank} W_{2}=2=n$ and the linear approximation of the form (3) is globally $\mathbb{R}$-controllable in the interval $[0,2]$. Let us observe that the cone $V_{2}^{c} \subset \mathbb{R}^{2}$ has the following form:

$$
\begin{aligned}
V_{2}^{c}= & \left\{x \in \mathbb{R}^{2}: x=A B u(0)+B u(1)\right. \\
= & {\left[\begin{array}{c}
1 \\
-1
\end{array}\right] u(0) } \\
& \left.+\left[\begin{array}{c}
-1 \\
1
\end{array}\right] u(1), u(0) \geq 0, u(1) \geq 0\right\} .
\end{aligned}
$$

Therefore $V_{2}^{c}=\mathbb{R}^{2}$ and $V_{2}^{c *}=\{0\}$. Hence, by Theorem 1 , the linear approximation of the form (3) is globally $U^{c}$-controllable in the interval $[0,2]$. Since $f(0,0)=0$ and the nonlinear function $\mathrm{f}$ is continuously differentiable near the origin, by Theorem 2 the nonlinear discrete system (10) is locally $U^{c 0}$-controllable in the interval $[0,2]$.

\section{Nonlinear 2-D Systems}

Consider the general nonlinear discrete 2-D system with constant coefficients described by the following difference equation:

$x(i+1, j+1)=f(x(i, j), x(i+1, j), x(i, j+1), u(i, j))$,

where $(i, j) \in \mathbb{Z}^{+} \times \mathbb{Z}^{+}, x(i, j) \in \mathbb{R}^{n}$ is the state vector at the point $(i, j), u(i, j) \in \mathbb{R}^{m}$ is the control vector at the point $(i, j)$, and $f: \mathbb{R}^{n} \times \mathbb{R}^{n} \times \mathbb{R}^{n} \times \mathbb{R}^{m} \rightarrow \mathbb{R}^{n}$ is a given function.

Let $U \subset \mathbb{R}^{m}$ be a given arbitrary set. The sequence of controls $u=\{u(i, j) ;(0,0) \leq(i, j), u(i, j) \in U\}$ is called an admissible sequence of controls. The set of all such admissible sequences of controls forms the so-called admissible set of controls. In the sequel, we shall also use the following notation: $\Omega^{0} \subset \mathbb{R}^{m}$ is a neighbourhood of zero, $U^{c} \subset \mathbb{R}^{m}$ is a closed convex cone with vertex at zero and $U^{c 0}=U^{c} \cap \Omega^{0}$.

The boundary conditions for the nonlinear difference equation (11) are given by

$$
x(i, 0)=x_{i 0} \in \mathbb{R}^{n}, \quad x(0, j)=x_{0 j} \in \mathbb{R}^{n},
$$

for $(i, j) \in \mathbb{Z}^{+} \times \mathbb{Z}^{+}$, where $x_{i 0}$ and $x_{0 j}$ are known vectors.

For the given boundary conditions (12) and for an arbitrary admissible sequence of controls, there exists a unique solution of the nonlinear difference equation (11), which can be computed by successive iterations.

Instead of the nonlinear 2-D system (11), we shall also consider the associated linear discrete 2-D system 
with constant coefficients described by the following difference equation:

$$
\begin{aligned}
x(i+1, j+1)= & A_{0} x(i, j)+A_{1} x(i+1, j) \\
& +A_{2} x(i, j+1)+B u(i, j),
\end{aligned}
$$

defined for $(i, j) \geq(0,0)$, where $A_{0}, A_{1}, A_{2}$ are constant $n \times n$-dimensional matrices and $B$ is an $n \times m$ dimensional constant matrix.

For the linear 2-D system (13) we can define the socalled transition matrix $A^{i, j}$ as follows (Kaczorek, 1985; 1993):

1. $A^{0,0}=I$ (the identity $n \times n$-dimensional matrix),

2. $A^{i, j}=0$ (the zero matrix) for $i<0$ or/and $j<0$,

3. $A^{i, j}=A_{0} A^{i-1, j-1}+A_{1} A^{i, j-1}+A_{2} A^{i-1, j}$ for $i, j=0,1,2, \ldots$.

Using the transition matrix $A^{i, j}$, we can express the solution $x(i, j)$ of the linear 2-D system (13) in the following compact form (Kaczorek, 1985; 1993):

$$
\begin{aligned}
x(i, j)= & A^{i-1, j-1}\left(A_{0} x(0,0)+B u(0,0)\right) \\
& +\sum_{r=1}^{r=i}\left(A^{i-r, j-1} A_{1} x(r, 0)\right. \\
& +A^{i-r-1, j-1}\left(A_{0} x(r, 0)+B u(r, 0)\right) \\
& +\sum_{s=1}^{s=j}\left(A^{i-1, j-s} A_{2} x(0, s)\right. \\
& +A^{i-1, j-s-1}\left(A_{0} x(0, s)+B u(0, s)\right) \\
& +\sum_{r=1}^{r=i}\left(\sum_{s=1}^{s=j} A^{i-r-1, j-s-1} B u(r, s)\right) .
\end{aligned}
$$

For the zero boundary conditions (i.e., $x(i, 0)=$ $x(0, j)=0)$, the solution $x(i, j)$ to (1) is given by (Kaczorek, 1995):

$$
\begin{aligned}
x(i, j) & =\sum_{r=0}^{r=j-1}\left(\sum_{s=0}^{s=i-1} A^{i-r-1, j-s-1} B u(r, s)\right) \\
& =W_{i j} u_{i j},
\end{aligned}
$$

where

$$
\begin{gathered}
W_{i j}=\left[\begin{array}{l:l:l:l}
A^{i-1, j-1} B & A^{i-2, j-1} B & \cdots & A^{0, j-1} B \\
& A^{i-1, j-2} B & \cdots & A^{1,0} B
\end{array}\right]
\end{gathered}
$$

and

$$
\begin{aligned}
& u_{i j}=\left[u^{T}(0,0)\left|u^{T}(1,0)\right| \cdots \mid u^{T}(i-1,0)\right. \\
& u^{T}(0,1): \cdots \mid u^{T}(i-2, j-1) \\
& \left.u^{T}(i-1, j-1)\right] \in U_{i j}=\underbrace{U \times U \times \cdots \times U}_{(i j-1) \text {-times }} .
\end{aligned}
$$

Moreover, let the cone $V_{i j}^{c} \subset \mathbb{R}^{n}$ denote the image of the cone $U_{i j}^{c}$ under the linear mapping $W_{i j}: \mathbb{R}^{(i j-1) m} \rightarrow$ $\mathbb{R}^{n}$, and let $V_{i j}^{c *} \subset \mathbb{R}^{n}$ denote the so-called polar cone defined as follows:

$$
V_{i j}^{c *}=\left\{x^{*} \in \mathbb{R}^{n}:\left\langle x^{*}, x\right\rangle \leq 0 \text { for all } x \in V_{i j}^{c}\right\} .
$$

For linear and nonlinear discrete 2-D systems it is possible to define many different concepts of controllability, in much the same way as for linear 2-D systems.

In the sequel, we shall concentrate on local and global $U$-controllabilities in a given rectangle $[(0,0),(p, q)]=\{(i, j):(0,0) \leq(i, j) \leq(p, q)\}$.

Definition 3. System (1) is said to be globally $U$ controllable in a given rectangle $[(0,0),(p, q)]$ if for the zero boundary conditions $x_{i 0}=0, i=0,1,2, \ldots, p$, $x_{0 j}=0, j=0,1,2, \ldots, q$ and every vector $x^{\prime} \in \mathbb{R}^{n}$ there exists an admissible sequence of controls $\{u(i, j) \in$ $U ;(0,0) \leq(i, j)<(p, q)\}$ such that the corresponding solution of (1) satisfies the condition $x(p, q)=x^{\prime}$.

Definition 4. The system (1) is said to be locally $U$ controllable in a given rectangle $[(0,0),(p, q)]$ if for the zero boundary conditions $x_{i 0}=0, i=0,1,2, \ldots, p$, $x_{0 j}=0, j=0,1,2, \ldots, q$ there exists a neighbourhood of zero $D \subset \mathbb{R}^{n}$ such that for every point $x^{\prime} \in D$ there exists an admissible sequence of controls $\{u(i, j) \in U$; $(0,0) \leq(i, j)<(p, q)\}$ such that the corresponding solution of (1) satisfies $x(p, q)=x^{\prime}$.

Of course, the same definitions are valid for linear discrete 2-D systems (13). For linear 2-D systems various controllability conditions are well known in the literature (see, e.g., Kaczorek, 1985; 1993; Klamka, 1991b).

Now, we shall recall fundamental criteria for global $U^{c}$-controllability, global $\mathbb{R}^{m}$-controllability and local $\Omega^{0}$-controllability in a given rectangle $[(0,0),(p, q)]$ for the linear 2-D system (13).

Theorem 3. (Klamka, 1994) The linear system (13) is globally $U^{c}$-controllable in the rectangle $[(0,0),(p, q)]$ if and only if the following two conditions hold:

$$
\begin{gathered}
\operatorname{rank} W_{p q}=n, \\
V_{p q}^{c *}=\{0\} .
\end{gathered}
$$


From Theorem 3 we obtain the necessary and sufficient condition for the global controllability with unconstrained controls stated in the following corollary:

Corollary 5. (Klamka, 1991b) The linear 2-D system (13) is globally $\mathbb{R}^{m}$-controllable in the rectangle $[(0,0),(p, q)]$ if and only if

$$
\operatorname{rank} W_{p q}=n \text {. }
$$

It is well known (Klamka, 1988b) that for sets $U$ containing zero as an interior point, local constrained controllability is equivalent to global unconstrained controllability.

Corollary 6. (Klamka, 1988b) The linear system (13) is locally $\Omega^{0}$-controllable in the rectangle $[(0,0),(p, q)]$ if and only if it is globally $\mathbb{R}^{m}$-controllable in the rectangle $[(0,0),(p, q)]$.

Corollary 6 follows directly from the well-known fact that the range of a bounded linear operator covers the whole space if and only if this operator transforms some neighbourhood of zero onto some neighbourhood of zero in the range space (Graves, 1950).

\section{Controllability Conditions for 2-D Nonlinear Systems}

In this section we shall formulate and prove sufficient conditions of local $U$-controllability in a given rectangle $[(0,0),(p, q)]$ and different sets $U$ for the nonlinear discrete $2-\mathrm{D}$ system (1).

It is generally assumed that

1. $f(0,0,0,0)=0$,

2. the function $f(x, y, z, u)$ is continuously differentiable with respect to all its arguments in some neighbourhood of zero in the product space $\mathbb{R}^{n} \times \mathbb{R}^{n} \times \mathbb{R}^{n} \times$ $\mathbb{R}^{m}$.

Taking into account Assumption 2, let us introduce the following notation for the partial derivatives of $f(x, y, z, u)$ :

$$
\begin{aligned}
& A_{0}=f_{x}^{\prime}(0,0,0,0), \quad A_{1}=f_{y}^{\prime}(0,0,0,0), \\
& A_{2}=f_{z}^{\prime}(0,0,0,0), \quad B=f_{u}^{\prime}(0,0,0,0),
\end{aligned}
$$

where $A_{0}, A_{1}, A_{2}$ are $n \times n$-dimensional constant matrices, and $B$ is an $n \times m$-dimensional constant matrix.

Therefore, using standard methods (Klamka, 1994), it is possible to construct a linear approximation of the nonlinear discrete 2-D system (1). This linear approximation is valid in some neighbourhood of zero in the product space $\mathbb{R}^{n} \times \mathbb{R}^{n} \times \mathbb{R}^{n} \times \mathbb{R}^{m}$, and is given by the linear difference equation (3) with the matrices $A_{0}, A_{1}, A_{2}, B$ defined above. The proofs of the main results are based on some lemmas from functional analysis concerning the socalled nonlinear covering operators (Graves, 1950; Robinson 1986).

Now, we are in a position to formulate and prove the main result on the local $U$-controllability in the rectangle $[(0,0),(p, q)]$ for the nonlinear discrete 2-D system (11).

Theorem 4. (Klamka, 1996). Suppose that $U^{c} \subset$ $\mathbb{R}^{m}$ is a closed convex cone with vertex at zero. Then the nonlinear discrete 2-D system (11) is locally $U^{c 0}$ controllable in the rectangle $[(0,0),(p, q)]$ if its linear approximation near the origin given by the difference equation (3) is globally $U^{c}$-controllable in the same rectangle $[(0,0),(p, q)]$.

Proof. The proof of the theorem is based on Lemma 1. Let our nonlinear operator $F$ transform the space of admissible control sequences $\{u(i, j):(0,0) \leq(i, j) \leq(p, q)\}$ into the space of solutions at the point $(p, q)$ for the nonlinear 2-D system (1). More precisely, the nonlinear operator $F: \mathbb{R}^{m} \times \mathbb{R}^{m} \times \cdots \times \mathbb{R}^{m} \rightarrow \mathbb{R}^{n}$ is defined as follows:

$$
\begin{aligned}
F\{u(0,0), u(0,1), u(1,0), u(1,1), \ldots, & u(p, q-1)\} \\
& =x(p, q),
\end{aligned}
$$

where $x(p, q)$ is the solution at the point $(p, q)$ of the nonlinear system (1) corresponding to an admissible control sequence $\{u(i, j):(0,0) \leq(i, j)<(p, q)\}$. The Fréchet derivative of the operator $F$ at zero, denoted by $\mathrm{d} F(0)$, is a bounded linear operator defined by

$$
\begin{array}{r}
\mathrm{d} F(0)\{u(0,0), u(0,1), u(1,0), u(1,1), \ldots, u(p, q-1)\} \\
=x(p, q),
\end{array}
$$

where $x(p, q)$ is the solution at $(p, q)$ of the linear system (13) corresponding to an admissible controls sequence $\{u(i, j):(0,0) \leq(i, j)<(p, q)\}$.

It should be stressed that the sequences of controls in (21) and (22) contain exactly $p q-1 m$-dimensional control vectors localized in the rectangle $[(0,0),(p, q)]$. However, it should be pointed out that the control vectors $u(p, j), j=0,1,2, \ldots, q$ and $u(i, q), i=0,1,2, \ldots, p$ do not influence the solution $x(p, q)$ (see the difference equations (11) and (13)). Since $f(0,0,0,0)=0$, for zero boundary conditions the nonlinear operator $F$ transforms zero into zero, i.e. $F(0)=0$. If the linear system (13) is globally $U^{c}$-controllable in the rectangle $[(0,0),(p, q)]$, then the image of the Fréchet derivative $\mathrm{d} F(0)$ covers the whole space $\mathbb{R}^{n}$. Therefore, by the result stated at the beginning of the proof, the nonlinear operator $F$ covers some neighbourhood of zero in the space $\mathbb{R}^{n}$. Hence, 
by Definition 2 , the nonlinear system (11) is locally $U^{c}$ controllable in the rectangle $[(0,0),(p, q)]$.

Corollary 7. Under the assumptions of Theorem 4 the nonlinear discrete control system (11) is locally $U^{c 0}$. controllable in the given rectangle $[(0,0),(p, q)]$ if (17) and (18) hold.

If the relations (17) and (18) hold, then by Theorem 1 the linear system (11) is globally $U^{c}$-controllable in the rectangle $[(0,0),(p, q)]$. Therefore, by Theorem 2 , the nonlinear system (11) is locally $U^{c 0}$-controllable in the same rectangle $[(0,0),(p, q)]$.

In the case when $U$ contains zero as an interior point, we have the following sufficient condition for the local constrained controllability of nonlinear 2-D systems:

Corollary 8. Let $0 \in \operatorname{int}(U)$. Then the nonlinear system (11) is locally $U$-controllable in the rectangle $[(0,0),(p, q)]$ if its linear approximation near the origin, given by the difference equation (13), is locally $U$ controllable in the same rectangle $[(0,0),(p, q)]$.

Consider the special case of nonlinear 2-D systems, namely the system described by the nonlinear difference equation

$$
\begin{aligned}
x(i+1, j+1)= & A_{0}(x(i, j)) x(i, j) \\
& +A_{1}(x(i+1, j)) x(i+1, j) \\
& +A_{2}(x(i, j+1)) x(i, j+1) \\
& +B(x(i, j) u(i, j)),
\end{aligned}
$$

where $A_{0}(x(i, j)), A_{1}(x(i+1, j)), A_{2}(x(i, j+1))$, $B(x(i, j))$ are nonlinear differentiable matrices of suitable dimensions.

In this case, the associated linear discrete 2-D system with constant coefficients is described by the following difference equation:

$$
\begin{aligned}
x(i+1, j+1)= & A_{0}^{\prime} x(i, j)+A_{1}^{\prime} x(i+1, j) \\
& +A_{2}^{\prime} x(i, j+1)+B^{\prime} u(i, j),
\end{aligned}
$$

where

$A_{0}^{\prime}=A_{0}(0), \quad A_{1}^{\prime}=A_{1}(0), \quad A_{2}^{\prime}=A_{2}(0), \quad B^{\prime}=B(0)$.

Theorem 5. Suppose that $U^{c} \subset \mathbb{R}^{m}$ is a closed convex cone with vertex at zero. Then the nonlinear discrete 2-D system (13) is locally $U^{c 0}$-controllable in the rectangle $[(0,0),(p, q)]$ if its linear approximation near the origin given by the difference equation (24) is globally $U^{c}$ controllable in the same rectangle $[(0,0),(p, q)]$.
Proof. The left-hand side of the nonlinear equation (23) satisfies all the assumptions stated for the nonlinear function $f(x, y, z, w)$. Moreover, the discrete 2-D system (24) is the corresponding linear approximation of the nonlinear discrete 2-D system (23) near the origin. Therefore, by Theorem 4, the result follows.

Consider the nonlinear 2-D system described by the following set of two difference equations:

$$
\begin{aligned}
x_{1}(i+1, j+1)= & x_{1}(i, j)+x_{1}^{2}(i, j)-x_{2}(i+1, j) \\
& +u(i, j)+u^{2}(i, j), \\
x_{2}(i+1, j+1)= & x_{1}^{2}(i+1, j)-x_{2}(i, j+1) \\
& +x_{2}^{2}(i, j+1)+u(i, j),
\end{aligned}
$$

defined in the rectangle $[(0,0),(2,2)]$. Hence $n=2$, $m=1$ and $p=q=2$. Let us additionally assume that $U^{c}=\{u \in \mathbb{R}: u \geq 0\}$, i.e., the admissible scalar controls $u(i, j)$ are non-negative.

Linear approximation of the nonlinear 2-D system (25) near the origin has the form (13) with the following matrices:

$$
\begin{aligned}
& A_{0}=\left|\begin{array}{cc}
1 & 0 \\
0 & 0
\end{array}\right|, \quad A_{1}=\left|\begin{array}{cc}
0 & -1 \\
0 & 0
\end{array}\right|, \\
& A_{2}=\left|\begin{array}{cc}
0 & 0 \\
0 & -1
\end{array}\right|, \quad B=\left|\begin{array}{l}
1 \\
1
\end{array}\right| .
\end{aligned}
$$

Therefore the $2 \times 4$-dimensional constant matrix $W_{p q}=$ $W_{22}$ has the following form:

$$
\begin{aligned}
& W_{22}=\left|\begin{array}{l:l:l:l}
A^{1,1} B & A^{0,1} B & A^{1,0} B & B
\end{array}\right| \\
& =\left|\begin{array}{l:l:l:l}
\left(A_{0}+A_{1} A_{2}+A_{2} A_{1}\right) B & A_{1} B & A_{2} B & B
\end{array}\right| \\
& =\left|\begin{array}{c:c:c:c}
2 & -1 & 0 & 1 \\
0 & 0 & -1 & 1
\end{array}\right|
\end{aligned}
$$

Hence $\operatorname{rank} W_{22}=2=n$ and the linear approximation of the form (13) is globally $\mathbb{R}$-controllable in the rectangle $[(0,0),(2,2)]$. Let us observe that the cone $V_{22}^{c} \subset \mathbb{R}^{2}$ has the form

$$
V_{22}=\left\{\begin{aligned}
x= & \left|\begin{array}{l}
x_{1} \\
x_{2}
\end{array}\right| \in \mathbb{R}^{2}: \\
x= & \left|\begin{array}{l}
2 \\
0
\end{array}\right| u(0,0)+\left|\begin{array}{c}
-1 \\
0
\end{array}\right| u(1,0) \\
& +\left|\begin{array}{c}
0 \\
-1
\end{array}\right| u(0,1)+\left|\begin{array}{c}
1 \\
1
\end{array}\right| u(1,1)
\end{aligned}\right\} .
$$


Therefore $V_{22}^{c}=\mathbb{R}^{2}$ and $V_{22}^{c *}=\{0\}$. Hence by Theorem 1 the linear approximation of the form (13) is globally $U^{c}$-controllable in the rectangle $[(0,0),(2,2)]$. Since $f(0,0,0,0)=0$ and the function $f$ is continuously differentiable near the origin, by Theorem 5 the nonlinear 2D system (25) is locally $U^{c 0}$-controllable in the rectangle $[(0,0),(2,2)]$.

\section{Conclusions}

In the present paper local constrained controllability problems for nonlinear finite-dimensional discrete 1-D and 2D systems with constant coefficients have been formulated and discussed. Using some mapping theorems from nonlinear functional analysis and linear approximation methods, various sufficient conditions for constrained controllability in bounded domains were derived and proved.

The paper extends the controllability conditions with unconstrained controls given in the literature to cover nonlinear discrete 1-D and 2-D systems with constrained controls. In the paper only one simple model of nonlinear discrete systems was considered. The results presented in Section 3 can be extended in many different directions. For example, it is possible to formulate and prove sufficient local controllability conditions for nonlinear discrete time-varying 1-D systems (Klamka, 1991; 1993; 1995). Moreover, similar controllability results can be derived for very general nonlinear discrete systems with several independent variables, the so-called $M$-D systems (Klamka, 1991), and for inifinite-dimensional discrete systems defined in Hilbert spaces (Klamka, 1995).

Moreover, in the present paper, the constrained controllability problem was considered only for one simple mathematical model of nonlinear 2-D systems. The results obtained can be extended in many different directions. For example, it is possible to formulate sufficient local controllability conditions for other mathematical models of nonlinear discrete 2-D systems (Kaczorek, 1985; 1993; Klamka, 1991b), for nonlinear discrete 2-D systems with variable coefficients (Klamka, 1991b) and for nonlinear discrete $M$-D systems. Moreover, similar constrained controllability results can be derived for very general $M$-D nonlinear discrete systems with variable coefficients defined in infinite-dimensional linear spaces (Klamka, 1988a).

\section{Acknowledgement}

This paper was supported by the State Committee for Scientific Research (KBN) in Poland under grant no. 8T11A 01819.

\section{References}

Graves L.M. (1950): Some mapping theorems. — Duke Math. J., Vol. 17, No.1, pp. 111-114.

Kaczorek T. (1985): Two-Dimensional Linear Systems. Berlin: Springer.

Kaczorek T. (1993): Linear Control Systems. — New York: Wiley.

Kaczorek T. (1995): U-reachability and U-controllability of 2D Roesser model. - Bull. Polish Acad. Sci. Tech. Sci., Vol. 43, No. 1, pp. 31-37.

Klamka J. (1988a): M-dimensional nonstationary linear discrete systems in Banach spaces. - Proc. 12-th IMACS World Congress, Paris, Vol. 4, pp. 31-33.

Klamka J. (1988b): Constrained controllability of 2-D linear systems. - Proc. 12-th IMACS World Congress, Paris, Vol. 2, pp. 166-169.

Klamka J. (1991a): Complete controllability of singular 2-D system. - Proc. 13-th IMACS World Congress, Dublin, pp. 1839-1840.

Klamka J. (1991b): Controllability of Dynamical Systems. Dordrecht: Kluwer.

Klamka J. (1992): Controllability of nonlinear 2-D systems. Bull. Polish Acad. Sci. Tech. Sci., Vol. 40, No. 2, pp. 125133.

Klamka J. (1993): Controllability of dynamical systems -A survey. - Arch. Contr. Sci., Vol. 2, No. 3, pp. 281-307.

Klamka J. (1994): Constrained controllability of discrete 2-D linear systems. - Proc. IMACS Int. Symp. Signal Processing, Robotics and Neural Networks, Lille, France, pp. 166169.

Klamka J. (1995): Constrained controllability of nonlinear systems. - IMA J. Math. Contr. Inf., Vol. 12, No. 2, pp. 245252.

Klamka J. (1996): Controllability of 2-D nonlinear systems. Proc. 2-nd World Congress Nonlinear Analysis, Athens, Greece, pp. 196-199.

Robinson S.M. (1986): Stability theory for systems of inequalities. Part II: Differentiable nonlinear systems. — SIAM J. Numer. Anal., Vol. 13, No. 4, pp. 1261-1275.

Received: 2 October 2001 